\title{
Technique of measurement of ultra-low resistance of current conductive junction of rail lines as the problem of states object identification
}

\author{
Tarasov E.M., Isaicheva A.G. \\ Samara State Transport University
}

\begin{abstract}
Systems of railway automation and remote control play an important role in transportation process ensuring the safety of train traffic, so it is necessary to eliminate failure and malfunctions of all its components and devices. Article deals with the principle of measuring of current conductive junctions rail lines, which allows to carry out the measurement and calculation of the resistance in a wide range of variation (from tens micro-ohm to unit $\mathrm{ohm})$. The presented mathematical models of informative signs can be in automated complexes of diagnostic resistance of current conductive junctions' rail trunk lines, industrial railways, tram road and underground rail.
\end{abstract}

Keywords: Rail lines, return tractional network ,connection of joints, resistance of current conductive junctions, automated complexes of diagnostic

Citation: Tarasov E.M., Isaicheva A.G. Technique of measurement of ultralow resistance of current conductive junction of rail lines as the problem of states object identification. Proceedings of Information Technology and Nanotechnology (ITNT-2015), CEUR Workshop Proceedings, 2015; 1490: 397-401. DOI: 10.18287/1613-0073-2015-1490-397-401

Automation and remote control devices are the main technical means ensuring automatic regulation and safety movement of trains, in which the sensitive element of the primary sensor of information on the state of control areas are rail lines. On electrified rail ways as return used the rail lines mainly consisting of rail links of length of 25 meters, at fish joints interconnected with tighten bolts and to equalize the resistance of junctions, apply copper connections of joints, cut set $70 \mathrm{~mm}^{2}$. In terms of circulation in the area of heavy trains is getting loosening of tightening bolts, a partial or complete break of connections of joints that leads to fluctuations in electrical joint resistance and it adversely effects the stability work of the track - classifiers of rails lines states. By taking the normal traffic control course into accounting it is necessary to minimize eliminate faults and malfunctions operated devices of automation and remote control, it becomes apparent the important condition to provide control of the electrical resistance of current conductive junctions (CCJ). This requirement is particularly important of diagnosis of CCJ failures, which make up $15 \%$ of the total flow of failures of systems of automation and remote control. 
Based on the resistance of the operated current conductive junction is within 60 $300 \times 10^{-6} \mathrm{Ohm}$, manual measurements require time, provide low accuracy and are not in heavy traffic. At the same time the resistance of the connection of joints can grow from $60 \times 10^{-6}$ to $0.4 \mathrm{Ohm}$ and reaches the latest comes the effect of break the rail line to the signal current, which leads to the appearance of information on the refusal of automation and remote control system [1,2].

In this regard, the development of control device value of resistance current conductive junctions is an urgent problem and requires the development of new principles of measurement of resistance junctions distributed in the area of control.

Nowadays it is apparent impossibility of direct remote sensing resistance current conductive junctions because of the low resistance value (tens micro-Ohm), their distribution and the large number - 200 current conductive junctions in the control area of $2500 \mathrm{~m}$.

Indirect measurement of resistance current conductive junctions must be carried out in the operation of systems of interval train control when the rail line (the sensitive element of the state sensor of rail lines) receives only the voltage source survey to determine their status: available, busy or faulty.

The technique of indirect measurement of resistance junctions in the low value in complex of control devices of rail lines state is that the first phase is to realize training of the decision function of calculator resistance CCJ on the training set of known resistance, characterized by a set of primary informative features predetermined coordinates and discrete number of resistance junctions in the range of possible values, in a second phase using a pre-trained decision function of calculating coordinates and resistance junction decision device at each time moment reliably detects the calculation results belonging to the concrete coordinate and resistance. And, in the regard, the determination of the resistance current conductive junctions, mainly due to the decision of problems of selection of informative features related to resistance CCJ and the synthesis of the rules as a base which will be the assignment the result of the calculation to the coordinate location of the junction and its resistance.

As for the defition of the resistance junction and its coordinates, as informative signs, conveniently use the input and outpt power rail quadripole characteristics, depending on the condition of rail line of control area, including the resistance value CCJ, especially the amplitude and phase of the voltage and current at the input rail line as well as the amplitude and phase voltages at the output of the rail lines. Then, a multitude of images generated at these signs, take the form:

$$
m_{j}=\left\{U_{1_{j}}, \varphi_{1_{j}}, I_{1_{j}}, \psi_{1_{j}}, U_{2_{j}}, \varphi_{2_{j}}\right\}, \quad j=1,2, \ldots, n,
$$

It is necessary take into account that the elementary quadripole rail line $\left[A_{r l}\right]$ contains a link of rail line with distributed parameters and elements of the current conductive junctions with lumped parameters which resistance must be measured. Therefore, portions of the rail line in equivalent circuit (Figure 1) [1], it is necessary to replace the cascade connection quadripole link of rail line $\left[A_{r}\right]$ and the quadripole current conductive junction $\left[A_{j}\right]$, like 


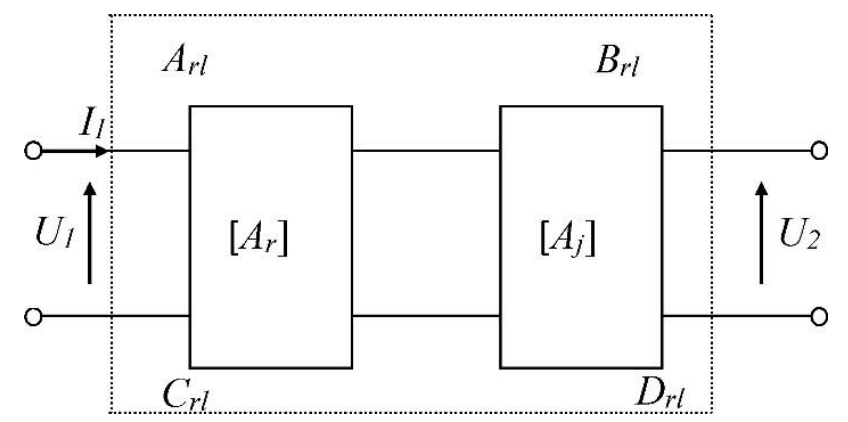

Fig. 1. The equivalent circuit rail quadripole current conductive junctions: $U_{1}, U_{2}$ - a complex value of the input and the output voltage of the elementary portion of the rail line; $I_{1}$ - complex current value at its output

Parameters of the generalized quadripole rail line, control section, formed by ncascade-connected quadripole link rail line and current conductive junction, defined by the expression

$$
A_{r l}=\bigvee_{i, j=1}^{n} A_{i j}^{r} A_{j i}^{l} \text {, }
$$

where $A_{i j}^{r}$ - quadripole rail link matrix; $A_{j i}^{l}$ - quadripole current conductive junction matrix; $n$-the number of rail links.

The rail line presents in the form of a cascade-connected n-quadripole rail lines with uniform distributed parameters and lumped current conductive junction which allows investigate the effect of measured parameter - current conductive junction on informative signs in the changing disturbances in the form of fluctuations resistance in the insulation of rail lines in a wide range.

Rail links, due to contact with the earth and the electromagnetic mutual effect are described by the equations of lines with distributed parameters [1], where

$$
\begin{aligned}
& A=D=\operatorname{ch} \gamma \ell, B=Z_{w} \operatorname{sh} g 1, C=\operatorname{sh} \gamma 1 / Z_{w}, \\
& \gamma=\alpha+j \beta=\sqrt{(r+j \omega L)(g+j \omega C)}, Z_{w}=\sqrt{\frac{r+j \omega L}{g+j \omega C}} .
\end{aligned}
$$

Rail quadripole matrix $\left[A_{r}\right]$ taking into account (2) and a current conductive junction $\left[A_{j}\right]$ take the form:

$$
\left[A_{r}\right]=\left[\begin{array}{ll}
A_{r} & B_{r} \\
C_{r} & D_{r}
\end{array}\right], \quad\left[A_{j}\right]=\left[\begin{array}{cc}
A_{j} & B_{j} \\
C_{j} & D_{j}
\end{array}\right] .
$$

In this case, the generalized matrix of the rail line portion control, as shown in Figure 1, is represented as: 


$$
\left\{\begin{array}{l}
A_{r l}=\operatorname{ch} \gamma l \cdot A_{j}+Z_{w} \operatorname{sh} \gamma l \cdot C_{j} \\
B_{r l}=\operatorname{ch} \gamma l \cdot B_{j}+Z_{w} \operatorname{sh} \gamma l \cdot D_{j} \\
C_{r l}=1 / Z_{w} \operatorname{sh} \gamma l \cdot A_{j}+\operatorname{ch} \gamma l \cdot C_{j} \\
D_{r l}=1 / Z_{w} \operatorname{sh} \gamma l \cdot B_{j}+\operatorname{ch} \gamma l \cdot D_{j}
\end{array}\right.
$$

Mathematical models of informative features (1) with regard to (2) and (4):

$U_{1}=\frac{E A_{r l} Z_{n}+B_{r l}}{\left(C_{r l} Z_{n}+D_{r l}\right) Z_{\mathrm{o}}+A_{r l} Z_{n}+B_{r l}}$

$I_{1}=\frac{E\left(C_{r l} Z_{n}+D_{r l}\right)}{\left(C_{r l} Z_{n}+D_{r l}\right) Z_{\mathrm{o}}+A_{r l} Z_{n}+B_{r l}}$

$$
U_{2}=\frac{E Z_{n}}{\left(C_{r l} Z_{n}+D_{r l}\right) Z_{\mathrm{o}}+A_{r l} Z_{n}+B_{r l}}
$$

where $E$ - source of interrogating signal of rail lines; $Z_{\mathrm{n}}, Z_{\mathrm{o}}$ - load resistance and limiter of the input current quadripole rail, respectively.

A crucial function of the calculator resistance $\mathrm{CCJ} Z_{i}(X)$ conveniently represented in the form of a polynomial of the form [3],

$$
Z_{i}(X)=C_{1} x_{1}+C_{2} x_{2}+\ldots+C_{m} x_{m}+C_{m+1}=C_{o}^{T} X+C_{m+1},
$$

where $x_{1}, x_{2}, \ldots x_{m}$ informative signs, $X$ - vector of informative signs, $C_{0}=\left(C_{1}, C_{2}, \ldots, C_{m}\right)^{T}$ transposed vector of coefficients.

In the process of constructing the decision function calculator in form (5), the main problem is to find the weight coefficients of polynomial, meet requirements the training calculator resistance current conductive junction for identifying discrete number resistance junctions in the range of possible value.

One way to solve this problem - the use of teaching methods [3,4]. Training calculator (determination of the coefficients of the polynomial calculator) can be achieved by solving the system of equations of condition [5] using the methods of interpolation theory. For this entire system of equations must be written in matrix form as $X C=Z$ [6], where $C=K^{-1} X^{T} Z$, where $X^{T}$ - transposed matrix $X ; K^{-1}$ - inverse matrix product, $K=X^{T} X$, which is equal to $\mathrm{K}^{-1}=\left(X^{T} X\right)^{-1}$.

The result is a single data recovery, mathematical modeling function having the minimum error in determining the resistance CCJ.

Obviously, the resulting polynomial must have tolerance "field" data not falling into the learning procedure function calculator, which can be verified on the control sample of experimental data. If the error of calculation of resistance decision function in the space of the "field" of data is significant and the results fall into the adjacent discrete resistances, then obviously you need to complicate the decision function and repeat the process of training calculator.

Considered the principle of measuring resistance current conductive junctions rail lines is implement, allows take the measurement and the calculation of the resistance in a wide range of variations (from tens micro-Ohm to units $\mathrm{Ohm}$ ) and can be used in automated diagnostic complexes resistance current conductive junctions rail trunk lines, industrial railways, tram road and underground line. 
The relative error of indirect measurement research of resistance current conductive junctions of training calculator is showed that the value of the relative error depends on the complexity of features and the number of signs in the functions of the calculator. So computer simulation resistance CCJ measurement restored function calculator is showed that the maximum relative error is observed in the determination of the resistance CCJ in the low resistance values $\delta\left(Z_{i \max }\right)=3.94 \%$, located closer to the source of the interrogation signal rail lines, and the minimum error $\delta\left(Z_{i \max }\right)=0.869 \%$ CCJ located at the output of the control.

\section{Reference}

1. Bryleev AM, Kravtsov Y, Shishlyakov AV. The theory, structure and operation of track circuits. M.: Transport, 1978; 344 p. [in Russian]

2. Dimitrenko IE. Technical diagnostics and auto control systems of railway automation and remote control. M.: Transport, 1986; 144 p. [in Russian]

3. Tu J, Gonzales R. Principles of recognition. M.: Mir, 1978; 412 p. [in Russian]

4. RF Patent №2173276 method for controlling the state of the rail line. EM Tarasov, Belonogov AS. Stated. 31.05.99. Publ. BI 2001, 25, V61L MCI 23/16.

5. Tarasov EM. The principle of separation of space images into classes decisive functions. Bulletin of Samara Scientific Center of Russian Academy of Sciences, Special Issue, Samara, 2003; 78-83.

6. Tarasov EM. Methods of determining the coefficients of the polynomial decision function. Vestnik Samara Municipal Institute of Management, Samara, 2012; 3(22): 48-56.

7. Tarasov EM, Yakobchuk AI. Generalized structure of the device monitoring and diagnostics. Vestnik Samara Municipal Institute of Management, Samara, 2011; 2(17): 164-170. 